\title{
Calcium Oxide: An Inexpensive Solid Support for Solvent Free Synthesis of Phosphorohydrazides and Thiophosphorohydrazide at Room Temperature
}

\author{
Nisha Kumari, Naresh Vyas, Hitendra.N.Karade and A.K.Gupta* \\ Defence R\&D Establishment, Jhansi Road, Gwalior 474002, Mp, India \\ arvindkumargupta2@rediffmail.com
}

\begin{abstract}
A simple, efficient, facile and surface mediated method for synthesis of hydrazides of organophosphorous acids has been developed from their corresponding chloridates using calcium oxides as a solid support at room temperature. The developed method gave the desired hydrazides in excellent yields (7592\%) and avoids the use of column chromatography.
\end{abstract}

Keywords: Calcium oxide; phosphorohydrazides; solvent free; Surface mediated; thiophosphorohydrazides.

\section{INTRODUCTION}

The hydrazides of organophosphorous acids (HOPAs) are important class of organophosphorous compounds (OPCs) and due to their synthetic applications as well as biological properties and their utility as building block of natural products has drawn the attention of organic, bioorganic and medicinal chemists. ${ }^{1}$ Extensive investigation have shown that these compounds exhibit fungicidal, acricidal, pesticidal, cytostatic, antibacterial, anti-microbial, anticonvulsant, anti-malarial, antituberculosis, antitumor activitiesand found useful ligands for transition metals. ${ }^{2-5}$ In literature there are several classical methods are reported for the synthesis of HOPAs. ${ }^{6-9}$ Among them the ToddAtherton reaction is the most prominent method in which dialkyl phosphites are treated with two moles of hydrazine and carbon tetrachloride under argon atmosphere. ${ }^{9}$ Although this method is a straightforward, but it suffers from several drawbacks, such as use of solvent, inert atmosphere, stringent reaction conditions, tedious work up, long reaction time and average yield. Other reported methods also involve similar kinds of drawbacks and cause difficulty in the isolation of the pure products or not environmentally friendly due to formation of side products and use of solvent. ${ }^{10}$

Modernly, there has been increasing accent on finding out low molecular weight recyclable environmentally safe materials or reagents. ${ }^{11}$ In this context, surface mediated reactions under solventfree conditions at room temperature are one of the most encouraging alternatives and have recently attracted attention due to legislative enforcement. ${ }^{12-14}$ There are several effects on a crystalline solid including heating, reduction of particle size, increase in surface area, generation of fresh surfaces, formation of defects, dislocations in crystal lattice, phase changes, mass transfer and save energy during grinding may have kinetic energy supplied by solid support. Due to wide utility of HOPAs and our interest to explore their synthetic potential, we have developed a new, efficient, and facile method for the synthesis of wide variety of HOPAs with divers structures bearing both $\mathrm{P}=\mathrm{S}$ and $\mathrm{P}=\mathrm{O}$ moiety.

\section{RESUlTS AND DisCUSSION}

Inspired with efficiency and simplicity of solid support, we focused on screening of inorganic solids to examine the effect for the synthesis of HOPAs. Therefore initially we prepared intermediates (monochloridate) by earlier reported methods. ${ }^{15}$ After having the intermediates in hand, we next performed various reactions of $N, N$-diethyl $O$-phenyl phosphoramidic chloride with hydrazine hydrate as model reaction in presence of different solid supports such as silica, sodium sulphate, anhydrous $\mathrm{MgSO}_{4}$, alumina neutral, alumina basic, $\mathrm{CaCO}_{3}, \mathrm{Na}_{2} \mathrm{CO}_{3}, \mathrm{CaO}, \mathrm{Na}_{2} \mathrm{CO}_{3}$-celite, and $\mathrm{CaO}$ celite at room temperature and other factor which governs the reaction were carefully investigated. All the reactions were monitored by TLC and ${ }^{31} \mathrm{P}$ NMR to find out the consumption of $N, N$-diethyl $O$ phenyl phosphoramidic chloride and formation of $N, N$-diethyl $O$-phenyl phosphoramidic hydrazide (Table-1).The results of the NMR analysis showed that $\mathrm{CaO}$ is superior in terms of quantitative 
conversion with lesser reaction time and exclusive formation of corresponding primary hydrazide. The optimized reaction conditions used in preparation of $N, N$-diethyl $O$-phenyl phosphoramidic hydrazide by using $\mathrm{CaO}$ as solid support was also compared by performing the same reaction conditions (except $\mathrm{CaO}$ ) with two widely used solvents(benzene \& dichloromethane). The results of this analysis showed that in absence of $\mathrm{CaO}$ even extended reaction time $(180 \mathrm{~min})$ had no significance enhancement in the yield (48-70\%). It is also notable that the use of $\mathrm{CaCO}_{3}$ is also found effective solid support but it took slightly higher time for completion of reaction. Other solid support either gave poor yield or posed difficulty in isolation of pure product.

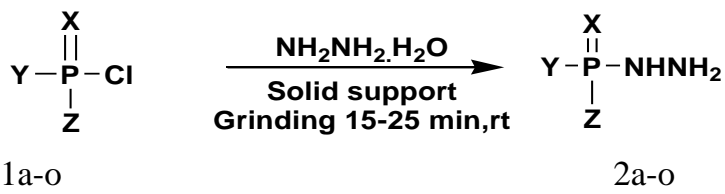

Scheme-1

$\mathrm{X}=\mathrm{O} \& \mathrm{~S}$

$\mathrm{Y}=\mathrm{OC}_{2} \mathrm{H}_{5}, \mathrm{O}^{i} \mathrm{C}_{3} \mathrm{H}_{7}, \mathrm{OC}_{6} \mathrm{H}_{5}, \mathrm{OCH}_{2} \mathrm{CH}_{2} \mathrm{OCH}_{3}$,

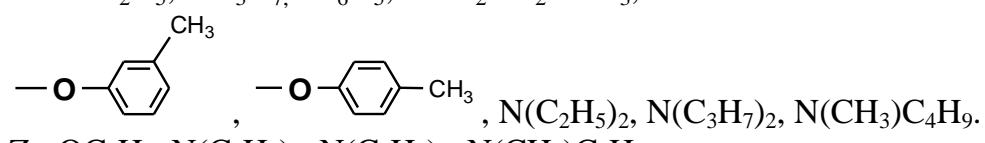

$\mathrm{Z}=\mathrm{OC}_{2} \mathrm{H}_{5}, \mathrm{~N}\left(\mathrm{C}_{2} \mathrm{H}_{5}\right)_{2}, \mathrm{~N}\left(\mathrm{C}_{3} \mathrm{H}_{7}\right)_{2}, \mathrm{~N}\left(\mathrm{CH}_{3}\right) \mathrm{C}_{4} \mathrm{H}_{9}$.

Table1. Conditions optimization for the synthesis of N,N-diethyl O-phenyl phosphoramidic hydrazide in presence of solid support at room temperature ${ }^{e}$

\begin{tabular}{|c|c|c|c|}
\hline Entry & Solid Support (0.02Mole) & $\begin{array}{l}\text { Reaction time } \\
\text { (min) }\end{array}$ & $\begin{array}{c}\text { Conversion }^{a} \\
(\%)\end{array}$ \\
\hline 1 & Nil & 120 & $48^{b}$ \\
\hline 2 & Nil & 180 & $70^{b}$ \\
\hline 3 & $\mathrm{SiO}_{2}$ & 60 & $52^{c}$ \\
\hline 4 & $\mathrm{Al}_{2} \mathrm{O}_{3}$ (basic) & 30 & 75 \\
\hline 5 & $\mathrm{Al}_{2} \mathrm{O}_{3}$ (neutral) & 30 & 35 \\
\hline 6 & $\mathrm{Na}_{2} \mathrm{CO}_{3}$ & 30 & 70 \\
\hline 7 & $\mathrm{Na}_{2} \mathrm{SO}_{4}$ & 30 & 50 \\
\hline 8 & $\mathrm{MgSO}_{4}$ & 30 & 52 \\
\hline 9 & $\mathrm{CaCO}_{3}$ & 45 & 90 \\
\hline 10 & $\mathrm{Na}_{2} \mathrm{CO}_{3}$-celite $\quad(1: 1$ molar ratio) & 30 & 75 \\
\hline 11 & CaO-celite $\quad$ (1:1 molar ratio) & 30 & 96 \\
\hline 12 & $\mathrm{CaO}$ & 60 & 100 \\
\hline 13 & $\mathrm{CaO}$ & 45 & 100 \\
\hline 14 & $\mathrm{CaO}$ & 20 & 100 \\
\hline 15 & $\mathrm{CaO}$ & 10 & $80^{d}$ \\
\hline 16 & $\mathrm{CaO}$ & 15 & 100 \\
\hline
\end{tabular}

${ }^{a}$ conversion based on ${ }^{31} P \mathrm{NMR}$ at $162 \mathrm{MHz}$ using $\mathrm{CDCl}_{3}$ except entry land 2 where $\mathrm{C}_{6} \mathrm{D}_{6}$ was used; ${ }^{b}$ reaction was performed in benzene solvent; reaction was found incomplete even in extended reaction time; other solvents were also attempted but no significant changes were observed; ${ }^{c}$ bis hydrazide was also observed as a parallel

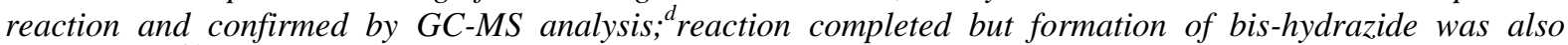
observed in ${ }^{31} P N M R ;{ }^{e}$ All the reactions were performed in mole ratio of 1:1 except entry 2 where reaction was performed in 1:2.

The results of the Table 1 showed that best results were obtained when reaction was carried out with 1: 1: 2 molar ratios of $N, N$-diethyl $O$-phenyl phosphoramidic chloride, hydrazine hydrate (99\%) using $\mathrm{CaO}$ as a solid support (entry 16). However, it was also observed that sequence of chemical addition plays crucial role in formation of the desired product and it is notable that $\mathrm{CaO}$ should be mixed with hydrazine hydrate, followed by addition of $N, N$-diethyl $O$-phenyl phosphoramidic chloride at room temperature and reaction mixture needs to be grinded as per time given in Table- 1 . The reverse addition of the chemical with solid support reduced the yield of the product, probably due to degradation of $N, N$-diethyl $O$-phenyl phosphoramidic chloride in corresponding acid. Further, it was also observed that by increasing the reaction time on $\mathrm{CaO}$, there is no significant change in the yield of products. After conditions optimization, the general applicability of newly developed method was 
Calcium Oxide: An Inexpensive Solid Support for Solvent Free Synthesis of Phosphorohydrazides and Thiophosphorohydrazide at Room Temperature

tested with a diversity of the structures of HOPAs and results of the reactions are summarized in Table 2. The compounds (2a-o) were characterized by spectroscopic techniques such as FT-IR, NMR, and GC/MS. The results are given in experimental section. However, FT-IR analysis showed the characteristic frequency for $\mathrm{P}=\mathrm{O}, \mathrm{P}=\mathrm{S}, \mathrm{P}-\mathrm{O}-\mathrm{C}, \mathrm{P}-\mathrm{N}-\mathrm{C}, \mathrm{NH}$ and $\mathrm{NH}_{2}$ linkage and were compared with literature values and found within the range. It is worth noting that the ${ }^{31} \mathrm{P} N M R$ signal of the monochloridate was disappeared with appearance of a new signal. After completion of reaction, the mixture was extracted with ether or dichloromethane to yield crude product with desirable purity without attempting crystallization and/or vacuum distillation (scheme-1).The recyclability of recovered $\mathrm{CaO}$ was checked four times in same reaction and found without loss of activity after washing the $\mathrm{CaO}$ with methanol and activating in an oven at $200^{\circ} \mathrm{C}$.

Table2: Synthesis of hydrazides of organophosphorous acids (2a-o) using calcium oxide as a solid support at room temperature

\begin{tabular}{|c|c|c|c|c|c|c|}
\hline $\begin{array}{l}\text { Sr. } \\
\text { No. }\end{array}$ & Substrate (1a-o) & Product (2a-o) & $\begin{array}{c}\text { Reaction } \\
\text { Time (min) } \\
\end{array}$ & $\begin{array}{c}\text { Physical } \\
\text { State } \\
\end{array}$ & $\begin{array}{l}\text { Isolated }^{a} \\
\text { Yield }(\%)\end{array}$ & $\begin{array}{c}{ }^{31} \mathbf{P} \text { NMR } \\
\left(\mathrm{CDCl}_{3}\right) \\
\end{array}$ \\
\hline $\mathrm{a}$ & & & 15 & $\begin{array}{l}\text { Viscous } \\
\text { liquid }\end{array}$ & 88 & 72.37 \\
\hline $\mathrm{b}$ & & & 20 & $\begin{array}{l}\text { Viscous } \\
\text { liquid }\end{array}$ & 82 & 77.04 \\
\hline $\mathrm{c}$ & & & 25 & $\begin{array}{l}\text { Viscous } \\
\text { liquid }\end{array}$ & 87 & 77.61 \\
\hline $\mathrm{d}$ & & & 20 & $\begin{array}{l}\text { Viscous } \\
\text { liquid }\end{array}$ & 82 & 78.13 \\
\hline $\mathrm{e}$ & & & 20 & $\begin{array}{l}\text { Viscous } \\
\text { liquid }\end{array}$ & 88 & 78.83 \\
\hline $\mathrm{f}$ & & & 15 & $\begin{array}{c}\text { liquid } \\
\text { Viscous }\end{array}$ & 92 & 13.38 \\
\hline $\mathrm{g}$ & & & 25 & $\begin{array}{l}\text { Viscous } \\
\text { liquid }\end{array}$ & 92 & 13.46 \\
\hline $\mathrm{h}$ & & & 25 & $\begin{array}{l}\text { Viscous } \\
\text { liquid }\end{array}$ & 82 & 13.56 \\
\hline $\bar{i}$ & & & 25 & $\begin{array}{l}\text { Viscous } \\
\text { liquid }\end{array}$ & 76 & 17.77 \\
\hline $\mathrm{j}$ & & & 15 & $\begin{array}{l}\text { Viscous } \\
\text { liquid }\end{array}$ & 75 & 16.25 \\
\hline $\mathrm{k}$ & & & 15 & $\begin{array}{l}\text { Viscous } \\
\text { liquid }\end{array}$ & 75 & 16.10 \\
\hline
\end{tabular}




\begin{tabular}{|c|c|c|c|c|c|c|}
\hline \hline $\mathrm{l}$ & & & 20 & $\begin{array}{c}\text { Viscous } \\
\text { liquid }\end{array}$ & 90 & 22.23 \\
\hline $\mathrm{m}$ & & & 25 & $\begin{array}{c}\text { Viscous } \\
\text { liquid }\end{array}$ & 85 & 22.79 \\
\hline $\mathrm{n}$ & & & 25 & $\begin{array}{c}\text { Viscous } \\
\text { liquid }\end{array}$ & 86 & 23.14 \\
\hline $\mathrm{o}$ & & & 25 & $\begin{array}{c}\text { Viscous } \\
\text { liquid }\end{array}$ & 88 & 22.73 \\
\hline
\end{tabular}

${ }^{a}$ The purity of the compounds was checked on silica TLC plate using chloroform/acetone mixture (8:2).

\section{EXPERIMENTAL}

An Agilent GC model 7820A instrument was used with flame ionization detector (FID). A capillary column (30m x $0.25 \mathrm{~mm}$ I.D-BP5) packed with 5\% phenyl and 95\% dimethyl polysiloxane (SGE) coated on fused silica was employed. The temperature of injection port and detector block were maintained at $280^{\circ} \mathrm{C}$ and $260^{\circ} \mathrm{C}$ respectively and the column oven was at programmed temperature profile started at $50^{\circ} \mathrm{C}$, ramped up to $280^{\circ} \mathrm{C}$ at $25^{\circ} \mathrm{C} / \mathrm{min}$. Nitrogen was used as a carrier gas (at a flow rate of $30 \mathrm{ml} / \mathrm{min}$ ). Air for FID was supplied at $300 \mathrm{ml} / \mathrm{min}$ and hydrogen at $30 \mathrm{ml} / \mathrm{min}$. In all analysis, $0.2 \mu \mathrm{l}$ sample were injected and peaks recorded on computerized data acquisition station. The GC-MS analysis were performed in EI $(70 \mathrm{eV})$ in full scan mode with an Agilent $6890 \mathrm{GC}$ equipped with a model 5973 mass selective detector (Agilent Technologies, USA). An SGE BPX5 capillary column with $30 \mathrm{~m}$ length $\times 0.32 \mathrm{~mm}$ internal diameter $\times 0.25 \mu \mathrm{m}$ film thickness was used at temperature program of $80^{\circ} \mathrm{C}(2 \mathrm{~min})-20^{\circ} \mathrm{C} / \mathrm{min}-280^{\circ} \mathrm{C}(3 \mathrm{~min})$. Helium was used as the carrier gas at a constant flow rate of $1.2 \mathrm{ml} / \mathrm{min}$. The samples were analyzed in splitless mode at injection temperature of $250^{\circ} \mathrm{C}$, EI source temperature $230^{\circ} \mathrm{C}$ and quadrupole analyzer at $150^{\circ} \mathrm{C}$. IR spectra were recorded on Bruker FT-IR spectrometer model alpha-T on $\mathrm{KBr}$ disk. ${ }^{1} \mathrm{H}$ and ${ }^{31} \mathrm{P}$ NMR spectra were recorded on Bruker DPX Avance FT-NMR in $\mathrm{CDCl}_{3}$ using tetramethylsilane as an internal standard for ${ }^{1} \mathrm{H}$ and $85 \% \mathrm{H}_{3} \mathrm{PO}_{4}$ as an external standard for ${ }^{31} \mathrm{P} N \mathrm{NMR}$ at 400 and $162 \mathrm{MHz}$ respectively.

All reagents and TLC plates were obtained from commercial sources (Sigma/Aldrich). However, $\mathrm{PCl}_{3}$ and $\mathrm{POCl}_{3}$, alcohols and amines were used after distillation. The purity of the products and progress of the reaction were monitored by thin layer chromatography (TLC) using commercial aluminumbacked silica gel sheets coated with silica gel $60_{\mathrm{F} 254}$. TLC spots were visualized under ultraviolet light or with iodine.Calcium oxide powder obtained from Himedia laboratories Pvt Limited and actvated by keeping in Isotemp vacuum oven model $281 \mathrm{~A}$ for two hour at $200^{\circ} \mathrm{c}$.

\section{1. $N, N$-Diethyl $O$-Phenyl Phosphoramidic Hydrazide (2f)}

In a typical experimental procedure, activated calcium oxide $(1.12 \mathrm{~g}, 0.01 \mathrm{~mol})$ was mixed with hydrazine hydrate $99 \%(0.16 \mathrm{~g}, 0.005 \mathrm{~mol})$ in a mortar and pestle. It was grinded for 2 minutes at room temperature and then $N, N$-diethyl $O$-phenyl phosphoramidic chloride $(1.18 \mathrm{~g}, 0.005 \mathrm{~mol})$ was added slowly. The reaction mixture was grinded for 15 minutes. The reaction was monitored by ${ }^{31} \mathrm{P}$ NMR after drawing few milligrams sample and extracted with diethyl ether. The results of ethereal solution analysis showed a signal appeared at $\delta 13.38$ and signal of $N, N$-diethyl $O$-phenyl phosphoramidic chloride $\delta 11.32$ disappeared. After completion of the reaction, the reaction mixture was extracted in diethyl ether and solid support was removed by filtration and the solvent was evaporated to afford pure $N, N$-diethyl $O$-phenyl phosphoramidic hydrazide $(92 \%, 1.06 \mathrm{~g})$ which was characterized by IR, GC-MS, ${ }^{1} \mathrm{H}$ and ${ }^{31} \mathrm{P}$ NMR. ${ }^{1} \mathrm{H}$ NMR $\left(400 \mathrm{MHz}, \mathrm{CDCl}_{3}\right): \delta 7.3(\mathrm{~m}, 5 \mathrm{H}, \mathrm{ArH}), 4.45(\mathrm{~d}, J=25.39 \mathrm{~Hz}$, $1 \mathrm{H}, \mathrm{NH}$ exchangeable), 3.49 (br s, $2 \mathrm{H}, \mathrm{NH}_{2}$ exchangeable), $3.20\left(\mathrm{~m}, J=7.79 \mathrm{~Hz}, 4 \mathrm{H}, \mathrm{CH}_{2}\right), 1.06(\mathrm{t}, J$ $\left.=7.97 \mathrm{~Hz}, 6 \mathrm{H}, \mathrm{CH}_{3}\right)$; IR KBr (neat): 3320, $3260(\mathrm{~N}-\mathrm{H}), 3140(\mathrm{~N}-\mathrm{H}), 2850(\mathrm{C}-\mathrm{H}), 1427(\mathrm{C}-\mathrm{N}), 1232$ $(\mathrm{P}=\mathrm{O}), \quad 1176 \quad(\mathrm{P}-\mathrm{N}-\mathrm{N}), \quad$ 1070, 1150 (P-N-C), 920(P-O-C) $\mathrm{cm}^{-1} ; \quad$ GC-MS (EI,\%): 244 $\left(\mathrm{M}+\mathrm{H}^{+}, 34.95\right), 243(78), 212(100), 77$ (51.96),72 (84.31). 
Calcium Oxide: An Inexpensive Solid Support for Solvent Free Synthesis of Phosphorohydrazides and Thiophosphorohydrazide at Room Temperature

Synthesis of $\boldsymbol{O}, \boldsymbol{O}$-Diethyl thiophosphoric hydrazide (2a). ${ }^{1} \mathrm{H}$ NMR $\left(400 \mathrm{MHz}, \mathrm{CDCl}_{3}\right) \delta 5.42(\mathrm{~d}, \boldsymbol{J}$ $27.34,1 \mathrm{H}, \mathrm{NH}$ exchangeable), $4.1\left(\mathrm{~m}, J=8.23 \mathrm{~Hz}, 4 \mathrm{H}, \mathrm{CH}_{2}\right), 3.12\left(\mathrm{br} \mathrm{s}, 2 \mathrm{H}, \mathrm{NH}_{2}\right.$ exchangeable), $1.28\left(\mathrm{t}, J=7.84 \mathrm{~Hz}, 6 \mathrm{H}, \mathrm{CH}_{3}\right)$; IR $\mathrm{KBr}$ (neat) $\mathrm{cm}^{-1}: 3316,3253(\mathrm{~N}-\mathrm{H}), 3142(\mathrm{~N}-\mathrm{H}), 2827(\mathrm{C}-\mathrm{H})$, $732(\mathrm{P}=\mathrm{S}), 1038(\mathrm{P}-\mathrm{O}-\mathrm{C})$; EIMS $(70 \mathrm{eV}) \mathrm{m} / \mathrm{z}$ (\% relative intensity): 185( $\left.\mathrm{M}+\mathrm{H}^{+}, 6.23\right), 184(100)$, 153(46.26).

$\mathrm{N}, \mathrm{N}$-Diethyl (3-methyl) $\boldsymbol{O}$-Phenyl thiophosphoramidic hydrazide (2b). ${ }^{1} \mathrm{H}$ NMR (400 $\mathrm{MHz}$, $\left.\mathrm{CDCl}_{3}\right): \delta 7.3(\mathrm{~m}, 4 \mathrm{H}, \mathrm{ArH}), 4.5(\mathrm{~d}, J=25.38 \mathrm{~Hz}, 1 \mathrm{H}, \mathrm{NH}$ exchangeable $), 3.52\left(\mathrm{br} \mathrm{s}, 2 \mathrm{H}, \mathrm{NH}_{2}\right.$ exchangeable), $3.34\left(\mathrm{~m}, J=7.74 \mathrm{~Hz}, 4 \mathrm{H}, \mathrm{CH}_{2}\right), 2.35\left(\mathrm{~s}, 3 \mathrm{H}, \mathrm{CH}_{3}\right), 1.10\left(\mathrm{t}, J=7.89 \mathrm{~Hz}, 6 \mathrm{H}, \mathrm{CH}_{3}\right)$; IR $\mathrm{KBr}$ (neat): 3324, $3258(\mathrm{~N}-\mathrm{H}), 3140(\mathrm{~N}-\mathrm{H}), 2848(\mathrm{C}-\mathrm{H}), 1422(\mathrm{C}-\mathrm{N}), 736(\mathrm{P}=\mathrm{S}), 1168(\mathrm{P}-\mathrm{N}-\mathrm{N})$, 1065, 1153 (P-N-C), 920 (P-O-C) cm ${ }^{-1}$; GC-MS (EI,\%): $274\left(\mathrm{M}+\mathrm{H}^{+}, 20.34\right), 273(49), 242(100), 172$ (31.9),137(24.23), 91 (44.66), 72 (84.31).

$\boldsymbol{N}, \boldsymbol{N}$-Dipropyl $\boldsymbol{O}$-phenyl thiophosphoramidic hydrazide (2c). ${ }^{1} \mathrm{H}$ NMR $\left(400 \mathrm{MHz}, \mathrm{CDCl}_{3}\right): \delta 7.37$ (m, $5 \mathrm{H}, \mathrm{ArH}), 4.28\left(\mathrm{~d}, J=27.86 \mathrm{~Hz}, 1 \mathrm{H}, \mathrm{NH}\right.$ exchangeable), 3.47 (br s, $2 \mathrm{H}, \mathrm{NH}_{2}$ exchangeable), 3.07 $\left(\mathrm{m}, J=8.57 \mathrm{~Hz}, 4 \mathrm{H}, \mathrm{CH}_{2}\right), 1.59\left(\mathrm{~m} J=8.77 \mathrm{~Hz}, 4 \mathrm{H}, \mathrm{CH}_{2}\right), 0.875\left(\mathrm{t}, J=7.87 \mathrm{~Hz}, 6 \mathrm{H}, \mathrm{CH}_{3}\right) ; \mathrm{IR} \mathrm{KBr}$ (neat): 3327, $3267(\mathrm{~N}-\mathrm{H}), 3147(\mathrm{~N}-\mathrm{H}), 2856(\mathrm{C}-\mathrm{H}), 1427(\mathrm{C}-\mathrm{N}), 738(\mathrm{P}=\mathrm{S}), 1174(\mathrm{P}-\mathrm{N}-\mathrm{N}), 1077$, 1157 (P-N-C), 925(P-O-C) cm ${ }^{-1}$; GC-MS (EI,\%): $288\left(\mathrm{M}+\mathrm{H}^{+}, \quad 6.37\right), 287(13.41), 256(100), 165$ (24.21), 108 (32.78)100(28.32).

$\boldsymbol{N}$-Methyl, $\boldsymbol{N}$-Butyl (4-methyl) $\boldsymbol{O}$-phenyl thiophosphoramidic hydrazide (2d). ${ }^{1} \mathrm{H}$ NMR (400 MHz, $\left.\left.\mathrm{CDCl}_{3}\right): \delta 7.32(\mathrm{~m}, 4 \mathrm{H}, \mathrm{ArH})\right), 4.22(\mathrm{~d}, J=29.32 \mathrm{~Hz}, 1 \mathrm{H}, \mathrm{NH}$ exchangeable $), 3.17\left(\mathrm{br} \mathrm{s}, 2 \mathrm{H}, \mathrm{NH}_{2}\right.$ exchangeable), $2.87\left(\mathrm{~m}, J=8.37 \mathrm{~Hz}, 2 \mathrm{H}, \mathrm{CH}_{2}\right), 2.39\left(\mathrm{~s} 3 \mathrm{H}, \mathrm{CH}_{3}\right), 2.27\left(\mathrm{~d}, J=8.36 \mathrm{~Hz}, 3 \mathrm{H}, \mathrm{N}-\mathrm{CH}_{3}\right)$, $1.453\left(\mathrm{~m}, J=8.28 \mathrm{~Hz}, 2 \mathrm{H}, \mathrm{CH}_{2}\right), 1.25\left(\mathrm{~m}, J=8.36 \mathrm{~Hz}, 2 \mathrm{H}, \mathrm{CH}_{2}\right), 0.95\left(\mathrm{t}, J=7.65 \mathrm{~Hz}, 3 \mathrm{H}, \mathrm{CH}_{3}\right)$; IR $\mathrm{KBr}$ (neat): 3325, $3215(\mathrm{~N}-\mathrm{H}), 3139(\mathrm{~N}-\mathrm{H}), 2826(\mathrm{C}-\mathrm{H}), 1434(\mathrm{C}-\mathrm{N}), 734(\mathrm{P}=\mathrm{S}), 1156(\mathrm{P}-\mathrm{N}-\mathrm{N})$,

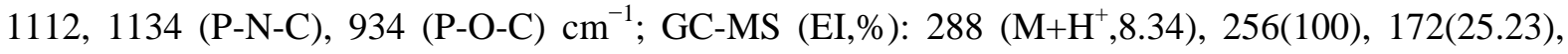
151(36.90), 108(45.76),86(56.43).

Bis-(, $\boldsymbol{N}$-Diethyl) thiophosphoramidic hydrazide (2e). ${ }^{1} \mathrm{H}$ NMR $\left(400 \mathrm{MHz}, \mathrm{CDCl}_{3}\right): \delta 3.87(\mathrm{~d}, J=$ $25.34 \mathrm{~Hz}, 1 \mathrm{H}, \mathrm{NH}$ exchangeable), 3.54 (br s, $2 \mathrm{H}, \mathrm{NH}_{2}$ exchangeable), $2.98(\mathrm{~m}, J=8.65 \mathrm{~Hz}, 8 \mathrm{H}$, $\left.\mathrm{CH}_{2}\right), 0.88\left(\mathrm{t}, J=8.78 \mathrm{~Hz}, 12 \mathrm{H}, \mathrm{CH}_{3}\right)$; IR KBr (neat): 3311, $3245(\mathrm{~N}-\mathrm{H}), 3122(\mathrm{~N}-\mathrm{H}), 2835(\mathrm{C}-\mathrm{H})$, 1410 (C-N), 730 (P=S), 1162 (P-N-N), 1057, 1125 (P-N-C), 913(P-O-C) cm ${ }^{-1}$; GC-MS (EI,\%): 239 $\left(\mathrm{M}+\mathrm{H}^{+}, 5.92\right), 238(78), 207(100), 137$ (24.50),72 (86).

$\boldsymbol{N}, \boldsymbol{N}$-Dipropyl (4-methyl) $\boldsymbol{O}$-phenyl phosphoramidic hydrazide (2g). ${ }^{1} \mathrm{H}$ NMR (400 $\left.\mathrm{MHz}, \mathrm{CDCl}_{3}\right)$ : $\delta 7.34(\mathrm{~m}, 4 \mathrm{H}, \mathrm{ArH}), 4.25(\mathrm{~d}, J=27.40 \mathrm{~Hz}, 1 \mathrm{H}, \mathrm{NH}$ exchangeable $), 3.46\left(\mathrm{br} \mathrm{s}, 2 \mathrm{H}, \mathrm{NH}_{2}\right.$ exchangeable), $2.96\left(\mathrm{~m}, J=8.58 \mathrm{~Hz}, 4 \mathrm{H}, \mathrm{CH}_{2}\right), 2.36\left(\mathrm{~s} 3 \mathrm{H}, \mathrm{CH}_{3}\right) 1.61\left(\mathrm{~m} \mathrm{~J}=8.76 \mathrm{~Hz}, 4 \mathrm{H}_{1} \mathrm{CH}_{2}\right)$, $0.867\left(\mathrm{t}, J=7.91 \mathrm{~Hz}, 6 \mathrm{H}, \mathrm{CH}_{3}\right)$; IR KBr (neat) : 3328, $3268(\mathrm{~N}-\mathrm{H}), 3148(\mathrm{~N}-\mathrm{H}), 2858(\mathrm{C}-\mathrm{H}), 1429$ (C-N), 1231 (P=O), 1181 (P-N-N), 1083, 1162 (P-N-C), 926 (P-O-C) cm ${ }^{-1}$; GC-MS (EI,\%): 286 $\left(\mathrm{M}+\mathrm{H}^{+}, 4.38\right), 285(28.10), 268$ (100),256 (48.21), 108(12.45), 91(29.43).

N-Methyl,N-Butyl (3- methyl) O-phenyl phosphoramidic hydrazide (2h). ${ }^{1} \mathrm{H}$ NMR (400 MHz, $\left.\left.\mathrm{CDCl}_{3}\right): \delta 7.3(\mathrm{~m}, 4 \mathrm{H}, \mathrm{ArH})\right), 4.32(\mathrm{~d}, J=29.32 \mathrm{~Hz}, 1 \mathrm{H}, \mathrm{NH}$ exchangeable $), 3.02\left(\mathrm{br} \mathrm{s}, 2 \mathrm{H}, \mathrm{NH}_{2}\right.$ exchangeable), $\left.2.93\left(\mathrm{~m}, J=8.38 \mathrm{~Hz}, 2 \mathrm{H}, \mathrm{CH}_{2}\right), 2.34\left(\mathrm{~s} 3 \mathrm{H}, \mathrm{CH}_{3}\right), 2.26\left(\mathrm{~d}, J=8.37 \mathrm{~Hz}, 3 \mathrm{H}, \mathrm{N}-\mathrm{CH}_{3}\right)\right)$, $1.34\left(\mathrm{~m}, J=8.45 \mathrm{~Hz}, 2 \mathrm{H}, \mathrm{CH}_{2}\right), 1.16\left(\mathrm{~m}, J=8.15 \mathrm{~Hz}, 2 \mathrm{H}, \mathrm{CH}_{2}\right), 0.912\left(\mathrm{t}, J=7.58 \mathrm{~Hz}, 3 \mathrm{H}, \mathrm{CH}_{3}\right) ; \mathrm{IR}$ $\mathrm{KBr}$ (neat) : 3319, $3213(\mathrm{~N}-\mathrm{H}), 3166(\mathrm{~N}-\mathrm{H}), 2846(\mathrm{C}-\mathrm{H}), 1424(\mathrm{C}-\mathrm{N}), 1204(\mathrm{P}=\mathrm{O}), 1147(\mathrm{P}-\mathrm{N}-\mathrm{N})$, 1115, 1123 (P-N-C), 945 (P-O-C) $\mathrm{cm}^{-1}$; GC-MS (EI,\%): $272\left(\mathrm{M}+\mathrm{H}^{+}, 12.21\right), 241(100), 108(45.48)$, 86(34.24).

$\boldsymbol{N}, \boldsymbol{N}$-Diproyl $\boldsymbol{O}$-methoxyethane phosphoramidic hydrazide(2i). ${ }^{1} \mathrm{H}$ NMR $\left(400 \mathrm{MHz}, \mathrm{CDCl}_{3}\right): \delta$ $3.95(\mathrm{~d}, J=19.47 \mathrm{~Hz}, 1 \mathrm{H}, \mathrm{NH}$ exchangeable $), 3.33$ (br s, $2 \mathrm{H}, \mathrm{NH}_{2}$ exchangeable $), 2.9(\mathrm{~m}, J=8.38$ $\left.\mathrm{Hz}, 4 \mathrm{H}, \mathrm{CH}_{2}\right), 1.63\left(\mathrm{~s}, 3 \mathrm{H}, \mathrm{CH}_{3}\right), 1.55\left(\mathrm{~m}, J=8.43 \mathrm{~Hz}, 4 \mathrm{H}, \mathrm{CH}_{2}\right), 1.26\left(\mathrm{t}, J=7.86 \mathrm{~Hz}, 2 \mathrm{H}, \mathrm{CH}_{2}\right), 1.23$ $\left(\mathrm{t}, J=7.89 \mathrm{~Hz}, 2 \mathrm{H}, \mathrm{CH}_{2}\right) 0.89\left(\mathrm{t}, J=7.88 \mathrm{~Hz}, 6 \mathrm{H}, \mathrm{CH}_{3}\right) ; \mathrm{IR} \mathrm{KBr}$ (neat) : 3343, $3256(\mathrm{~N}-\mathrm{H}), 3135(\mathrm{~N}-$ $\mathrm{H}), 2952(\mathrm{C}-\mathrm{H}), 1447(\mathrm{C}-\mathrm{N}), 1238(\mathrm{P}=\mathrm{O}), 1064,1160(\mathrm{P}-\mathrm{N}-\mathrm{C}) \mathrm{cm}^{-1}$; GC-MS (EI,\%): 254 $\left(\mathrm{M}+\mathrm{H}^{+}, 4.45\right), 25(65.34), 222(57.23), 153(100), 59(95)$.

$\boldsymbol{O}$-Isopropyl $\boldsymbol{N}$-Methyl, $\mathbf{N}$-Butyl phosphoramidic hydrazide(2j). ${ }^{1} \mathrm{H}$ NMR $\left(400 \mathrm{MHz}, \mathrm{CDCl}_{3}\right): \delta$ $4.62(\mathrm{~m}, J=19.51 \mathrm{~Hz}, 1 \mathrm{H}, \mathrm{CH}), 3.94\left(\mathrm{~d}, J=19.34 \mathrm{~Hz}, 1 \mathrm{H}, \mathrm{NH}\right.$ exchangeable), 3.31 (br s, $2 \mathrm{H}, \mathrm{NH}_{2}$ 
exchangeable), $3.0\left(\mathrm{~m}, J=8.34 \mathrm{~Hz}, 2 \mathrm{H}, \mathrm{CH}_{2}\right), 2.68\left(\mathrm{~d}, J=8.46 \mathrm{~Hz}, 3 \mathrm{H}, \mathrm{N}-\mathrm{CH}_{3}\right) 1.53(\mathrm{~m}, J=8.44 \mathrm{~Hz}$, $\left.2 \mathrm{H}, \mathrm{CH}_{2}\right), 1.43\left(\mathrm{~m}, J=8.34 \mathrm{~Hz}, 2 \mathrm{H}, \mathrm{CH}_{2}\right), 1.49\left(\mathrm{~d}, J=6.47 \mathrm{~Hz}, 6 \mathrm{H}, \mathrm{CH}_{3}\right), 0.93\left(\mathrm{t}, \mathrm{J}=7.88 \mathrm{~Hz}, 3 \mathrm{H}, \mathrm{CH}_{3}\right)$; IR KBr (neat) : 3329, $3266(\mathrm{~N}-\mathrm{H}), 3144(\mathrm{~N}-\mathrm{H}), 2927(\mathrm{C}-\mathrm{H}), 1438(\mathrm{C}-\mathrm{N}), 1235(\mathrm{P}=\mathrm{O}), 1084,1150$ (P-N-C) $\mathrm{cm}^{-1}$; GC-MS (EI,\%): $224\left(\mathrm{M}+\mathrm{H}^{+}\right.$,5.45), 192(100), 135(10.34).

$\boldsymbol{O}$-Isopropyl $\boldsymbol{N}, \boldsymbol{N}$-Diproylphosphoramidic hydrazide (2k). ${ }^{1} \mathrm{H}$ NMR $\left(400 \mathrm{MHz}, \mathrm{CDCl}_{3}\right): \delta 4.63$ (m, $J=19.54 \mathrm{~Hz}, 1 \mathrm{H}, \mathrm{CH}), 3.97(\mathrm{~d}, J=19.36 \mathrm{~Hz}, 1 \mathrm{H}, \mathrm{NH}$ exchangeable $), 3.3\left(\mathrm{br} \mathrm{s}, 2 \mathrm{H}, \mathrm{NH}_{2}\right.$ exchangeable), $2.9\left(\mathrm{~m}, J=8.33 \mathrm{~Hz}, 4 \mathrm{H}_{2} \mathrm{CH}_{2}\right), 1.55\left(\mathrm{~m}, J=8.45 \mathrm{~Hz}, 4 \mathrm{H}_{1} \mathrm{CH}_{2}\right), 1.3(\mathrm{~d}, J=6.47 \mathrm{~Hz}$, $\left.6 \mathrm{H}, \mathrm{CH}_{3}\right), 0.88\left(\mathrm{t}, \mathrm{J}=7.87,6 \mathrm{H}, \mathrm{CH}_{3}\right) ; \mathrm{IR} \mathrm{KBr}($ neat $): 3325,3264(\mathrm{~N}-\mathrm{H}), 3143(\mathrm{~N}-\mathrm{H}), 2925(\mathrm{C}-\mathrm{H})$, $1436(\mathrm{C}-\mathrm{N}), 1236(\mathrm{P}=\mathrm{O}), 1084,1150(\mathrm{P}-\mathrm{N}-\mathrm{C}) \mathrm{cm}^{-1}$; GC-MS (EI,\%): $238\left(\mathrm{M}+\mathrm{H}^{+}, 4.45\right), 237(8.34)$, 166(100), 100(16.26).

Bis-( $N, N$-Diethyl) phosphoramidic hydrazide (2l). ${ }^{1} \mathrm{H}$ NMR $\left(400 \mathrm{MHz}, \mathrm{CDCl}_{3}\right): \delta 3.74(\mathrm{~d}, J=$ $25.28 \mathrm{~Hz}, 1 \mathrm{H}, \mathrm{NH}$ exchangeable), $3.45\left(\mathrm{br} \mathrm{s}, 2 \mathrm{H}, \mathrm{NH}_{2}\right.$ exchangeable $), 2.89(\mathrm{~m}, J=8.56 \mathrm{~Hz}, 8 \mathrm{H}$, $\left.\mathrm{CH}_{2}\right), 0.85\left(\mathrm{t}, J=8.73 \mathrm{~Hz}, 12 \mathrm{H}, \mathrm{CH}_{3}\right)$; IR KBr (neat) : 3315, $3255(\mathrm{~N}-\mathrm{H}), 3132(\mathrm{~N}-\mathrm{H}), 2845(\mathrm{C}-\mathrm{H})$, $1420(\mathrm{C}-\mathrm{N}), 1230(\mathrm{P}=\mathrm{O}), 1172(\mathrm{P}-\mathrm{N}-\mathrm{N}), 1067,1135$ (P-N-C), 923(P-O-C) $\mathrm{cm}^{-1}$; GC-MS (EI,\%): 223 $\left(\mathrm{M}+\mathrm{H}^{+}, 4.95\right), 222(78), \mathrm{s}$ 191(46.07), 120 (24.50),72 (100).

$N, N$-Diethyl $N, N$-Diproyl phosphoramidic hydrazide (2m). ${ }^{1} \mathrm{H}$ NMR $\left(400 \mathrm{MHz}, \mathrm{CDCl}_{3}\right): \delta 3.84(\mathrm{~d}$, $J=29.33 \mathrm{~Hz}, 1 \mathrm{H}, \mathrm{NH}$ exchangeable), 3.29 (br s, $2 \mathrm{H}, \mathrm{NH}_{2}$ exchangeable), $3.18(\mathrm{~m}, J=8.33 \mathrm{~Hz}, 8 \mathrm{H}$, $\mathrm{CH} 2), 1.67(\mathrm{~m}, J=8.31 \mathrm{~Hz}, 4 \mathrm{H}, \mathrm{CH} 2), 1.12\left(\mathrm{t}, J=7.66 \mathrm{~Hz}, 6 \mathrm{H}, \mathrm{CH}_{3}\right), 1.10(\mathrm{t}, J=7.53$ $\left.\mathrm{Hz}, 6 \mathrm{H}, \mathrm{CH}_{3}\right)$; IR $\mathrm{KBr}$ (neat) : 3319, $3259(\mathrm{~N}-\mathrm{H}), 3138(\mathrm{~N}-\mathrm{H}), 2837(\mathrm{C}-\mathrm{H}), 1437(\mathrm{C}-\mathrm{N}), 1236(\mathrm{P}=\mathrm{O})$, 1162 (P-N-N), 1143, 1064 (P-N-C), 924(P-O-C) $\mathrm{cm}^{-1}$; GC-MS (EI,\%): $251\left(\mathrm{M}+\mathrm{H}^{+}, 12.23\right), 250(58)$, 219(100), 100(56.26), 72(46.28).

$\boldsymbol{N}, \boldsymbol{N}$-Dipropyl $\boldsymbol{N}$-Methyl, $\boldsymbol{N}$-Butyl Phosphoramidic hydrazide (2n). ${ }^{1} \mathrm{H}$ NMR $\left(400 \mathrm{MHz}, \mathrm{CDCl}_{3}\right): \delta$ $3.794\left(\mathrm{~d}, J=29.54 \mathrm{~Hz}, 1 \mathrm{H}, \mathrm{NH}\right.$ exchangeable), 3.45 (br s, $2 \mathrm{H}, \mathrm{NH}_{2}$ exchangeable), 2.94 (m, $J=8.35$ $\left.\left.\mathrm{Hz}, 6 \mathrm{H}, \mathrm{CH}_{2}\right), 2.64\left(\mathrm{~d}, J=8.48 \mathrm{~Hz}, 3 \mathrm{H}, \mathrm{N}-\mathrm{CH}_{3}\right)\right), 1.58\left(\mathrm{~m}, J=8.34 \mathrm{~Hz}, 6 \mathrm{H}, \mathrm{CH}_{2}\right), 1.34(\mathrm{~m}, J=8.18$ $\left.\mathrm{Hz}, 2 \mathrm{H}, \mathrm{CH}_{2}\right), 0.93\left(\mathrm{t}, J=7.59 \mathrm{~Hz}, 6 \mathrm{H}, \mathrm{CH}_{3}\right), 0.87\left(\mathrm{t}, J=7.50 \mathrm{~Hz}, 3 \mathrm{H}, \mathrm{CH}_{3}\right) ; \mathrm{IR} \mathrm{KBr}$ (neat) : 3313, $3243(\mathrm{~N}-\mathrm{H}), 3133(\mathrm{~N}-\mathrm{H}), 2860(\mathrm{C}-\mathrm{H}), 1413(\mathrm{C}-\mathrm{N}), 1238(\mathrm{P}=\mathrm{O}), 1171(\mathrm{P}-\mathrm{N}-\mathrm{N}), 1143,1062(\mathrm{P}-\mathrm{N}-\mathrm{C})$,

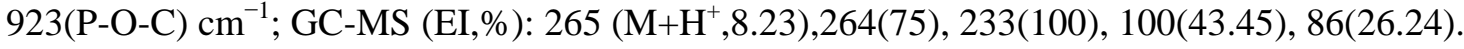

Bis-( $N$-Methyl, $N$-Butyl) Phosphoramidic hydrazide (2o). ${ }^{1} \mathrm{H}$ NMR (400 MHz, $\left.\mathrm{CDCl}_{3}\right): \delta 4.32$ (d, $J$ $=29.34 \mathrm{~Hz}, 1 \mathrm{H}, \mathrm{NH}$ exchangeable $), 3.25\left(\mathrm{br} \mathrm{s}, 2 \mathrm{H}, \mathrm{NH}_{2}\right.$ exchangeable $), 2.96(\mathrm{~m}, J=8.43 \mathrm{~Hz}$, $\left.\left.4 \mathrm{H}, \mathrm{CH}_{2}\right), 2.36\left(\mathrm{~d}, J=8.47 \mathrm{~Hz}, 6 \mathrm{H}, \mathrm{N}-\mathrm{CH}_{3}\right)\right), 1.45\left(\mathrm{~m}, J=8.44 \mathrm{~Hz}, 4 \mathrm{H}, \mathrm{CH}_{2}\right), 1.25(\mathrm{~m}, J=8.16 \mathrm{~Hz}$, $\left.4 \mathrm{H}, \mathrm{CH}_{2}\right), 0.96\left(\mathrm{t}, J=7.54 \mathrm{~Hz}, 6 \mathrm{H}, \mathrm{CH}_{3}\right)$; IR KBr (neat) : 3323, $3219(\mathrm{~N}-\mathrm{H}), 3146(\mathrm{~N}-\mathrm{H}), 2845(\mathrm{C}-$ H), $1425(\mathrm{C}-\mathrm{N}), 1208(\mathrm{P}=\mathrm{O}), 1149$ (P-N-N), 1105, 1113 (P-N-C), 942 (P-O-C) cm ${ }^{-1}$; GC-MS (EI,\%): $251\left(\mathrm{M}+\mathrm{H}^{+}, 34.21\right), 250(82), 219(100), 86(56.26)$.

\section{Conclusion}

In conclusion, we have developed a rapid, efficient and facile solvent free surface mediated method for the synthesis of HOPAs in special context of Bis-( $N, N$-dialkyl) phosphoramidic hydrazides, $O$-aryl $N, N$ - dialkyl phosphoramidic hydrazide and $N, N$-dialkylamino alkyl phosphonohydrazide and their thio analogues in excellent yields. The main advantage of this method is that reactions were clean and had operational simplicity. The method becomes more attractive for organic chemists as column chromatography was not required to purify the products. The synthetic applications of these hydrazides are in progress and will be published shortly.

\section{ACKNOWLEDGEMENTS}

We thank Dr.Lokendra Singh, Director, DRDE,Gwalior, and Dr. D. K. Dubey Associate Director for his keen interest, encouragement and critical comments. The authors also thank to Rajesh Kinkar for his technical assistance and NMR analysis. Authors express their gratitude to University Grant Commission (New Delhi) for financial support.

\section{REFERENCES}

[1] (a) Hildebrand, R.; The Role of Phosphonates in Living Systems; CRC Press: Boca Raton,1983;(b) John, R.Van .Wazer., Phosphorus and its Compounds; Interscience, New York, 1961,Vol. II.;(c) Engel, R.; Chem. Rev.1977, 77, 349;(d) Corbridge, D. E. C. Studies in Inorganic Chemistry 10, Phosphorus An outline of its Chemistry Biochemistry and Technology, Elsevier Science Publishing Company: New York. 
Calcium Oxide: An Inexpensive Solid Support for Solvent Free Synthesis of Phosphorohydrazides and Thiophosphorohydrazide at Room Temperature

[2] (a) Melnkov, N.N.; Krylova, T.P.; Valdimorova, I.L. Zhur.Obshch.Khim. 1973, 43, 1646;(b) Blair, E.H. US P.2 855 424, 1958, Chem.Abs. 1959, 53, 4211;(c) Kornoukhova, M.V.; Lomakina, V.I.; Mandelbaum, Y.A. Zhur.Obshch.Khim. 1971, 41, 2004;(e) Farber, L. US P.3 576 916, 1971, Chem.Abs. 1971, 75, 36 388;(f) Cremlyn, R.J.W.; David, J.; Kishore, N. Pestic.Sci. 1974, 5, 667;(g) Shandruk, M.I.; Yanchuk, N.I.; Grekov, A.P. Zhur.Obshch.Khim. 1973, 43, 2198;(h) Englin, M.A.; Filatov, A.S.; Fraer, Z.I.; Promonenkov, V. K.; Ivin, S.Z. Zhur.Obshch.Khim. 1968, 38, 869;(i) Grapov, A.F.; Mikhailova, O.B.; Razvodovskya, L. V.; Melnikov, N.N.; Zhur.Obshch.Khim. 1971, 41, 1441.

[3] (a) Fest, C.; Schmidt, K. J. The Chemistry of organophosphorus pesticides, Springer Verlag, Berlin 1982;(b) Hinkle, P.; McCarty, R. Y. Sci. Amer. 1978,104, 238;(c) Eto, M.; Organophosphorus Pesticides: Organic and Biological chemistry; CRC Press: USA, 1974; 18.

[4] Gholivand, K.; Hosseini, Z.; Farshadian, S.; Manesh H. N., Eur. J. Med. Chem. 2010,45 5130.

[5] (a) Katti, K. V.; Ge, Y. W.; Singh, P. R.; Date, S. V.; Barnes,C. L. Organometallics. 1994, 13, 541;(b) Singh, P. R.; Jimenez, H.; Katti, K. V.; Volkert, W. A.; Barnes, C. L. Inorg. Chem. 1994, 33,736;(c) Wang, M. W.; Volkert, E. W.; Singh, P. R.; Katti, K. K.; Lusiak,P.; Katti K. V. ; Barnes, C. L. Inorg. Chem. 1994, 33, 1 184;(d) Reddy, V. S.; Katti, K. V.; Barnes, C. L. Chem. Ber. 1994, 127,979.

[6] (a) Ephraim, F.; Sackheim, M. Chem Ber. 1911, 44, 3416;(b) Autenrieth, W.; Meyer, W. Chem.Ber. 1925, 58, 848;(c) Audrieth, L. F.; Smith, W. C. J. Org. Chem. 1953, 20, 1288;(d) Smith, W. C.; Gher, R.; Audrieth, L. F. J. Org. Chem. 1953, 21, 1288;(e) Melnikov, N.N.; Zenkevich, A.G. Zhur.Obshch.Khim. 1955, 25, 828;(f) Kennard, K.C.; Hamilton, C.S. J.Amer.Chem.Soc, 1955, 77, 1156.

[7] (a) Smith, W.C.; Gher, R.; Andrieth, L.E. J.Org.Chem.1956, 21, 113;(b) Sambeth, J.; BeckeGoehring, M. Angew.Chem.1958, 70, 594;(c) Kreuzkamp, N.; Schindler, H. Arch.Pharm. 1960,293, 296;(d) Klement, R.; Knollmüller, K. O. Chem. Ber. 1961,94, 1457;(e) Klement, R.; Knollmuller, K.O. Chem.Ber. 1960,93, 1088.

[8] (a)H.Tolkmith, J. Amer. Chem. Soc. 1962,84, 2097; (b) Jan, H.; Vis.; Meinke, Phyllis. Can. J. Chem. 1969, 47, 1581; (c) Melnikov, N.N.; Mikhailova, O.B.; Grapov, A.F. Zhur.Obshch.Khim. 1968, 38, 2099;(d) Shandruk, M.I.; Yanchuk, N.I.; Grekov, A.P. Zhur.Obshch.Khim.1973, 43, 2194;(e) Konecny,V.; Kovac, S. Chem.Papers. 1986, 40,679;(f) Katti, K. V.; Reddy, V.S.; Singh, P. R. Chem.Soc.Rev.1995;(g)Kavita.; Joshi, P.; Sharma, N.; Joshi, Y. C. Can. J. Chem. 2010,88, 1034.

[9] (a) Le Corre, S. S.; Berchel, M.; Couthon-Gourves, H.; Haelters, J.P.; Jaffres, P.A. Beil.J. Org. Chem. 2014, 10, 1166;(b) Klement, R. ; Knollmüller, K. O. Chem. Ber. 1960,93, 834.

[10] (a) Zwierzak, A.; Sulewska, A. Synthesis. 1976,12, 835.(b) Cremlyn, R.J.W.; Dewhurst, B.B.; Wakeford, D.H.; J.Chem.Soc, 1971, 3011;(c) Michaelis, A. Annalen, 1903, 326, 197;(d) Ovrutskii, V.M.; Kuzmenko, I.I., Protsenko, L.D. Zhur.Obshch.Khim. 1977, 47, 307.

[11] (a) Sathe, M.; Gupta, A. K.; Kaushik, M. P. Tetrahedron Lett. 2006, 47, 3107;(b) Acharya, J.; Gupta, A. K.; Kaushik, M. P. Tetrahedron Lett.2005, 46, 5293;(c) Acharya, J.; Shakya, P. D.; Pardasani, D.; Palit, M.; Dubey, D. K.; Gupta, A. K. J. Chem. Res. 2005, 3, 194;(d) Gupta, A. K.; Dubey, D. K.; Kaushik. M. P. Phosphorus, Sulfur, and Silicon. 2008,183,1641.

[12] (a) Tanaka, K. Solvent-Free Organic Synthesis, Wiley-VCH: Weinheim,2003;(b) Gedye, R. J.; Westaway, K.; Ali, H.; Baldisera, L.; Laberge, L.; Rousell, J. Tetrahedron Lett. 1986,27, 279;(c) Tanaka,K.; Toda,F. Chem.Rev.2000,100,1025;(d) Verma,R.S. Green Chem.1999,1,43.

[13] (a) Giguere, R. J.; Bray, T. L.; Duncan, S. M.; Majetich, G. Tetrahedron Lett. 1986, 27, 4945;(b) Abramovitch, A. Org. Prep. Proc. Int. 1991, 23, 685;(c) Migos, D. M. P.; Baghurst, D. R. Chem. Soc. Rev. 1991, 20, 1;(d) Caddick, S. Tetrahedron, 1995,51, 10403.

[14] (a) Loupy, A.; Petit, A.; Ramdiani, M.; Yvanaeff, C.; Majdoub, M.; Labiad, B.; Villemin, D. Can. J. Chem. 1993,71, 90;(b) Baezza, A.; Najera, C.; Gracia, R.; Sansano, J. M. Synthesis, 2005, 16, 2787.

[15] Le Corre, SS.; Berchel, M.; Belmadi, N.; Denis, C.; Org.Biomol.Chem,2014,12,1463. 


\section{AUTHORS' BIOGRAPHY}

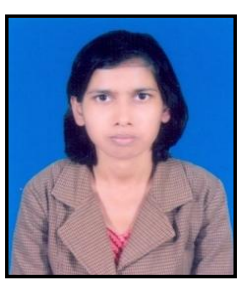

Ms Nisha Kumari, obtained her M.Sc. (Organic Chemistry) from the Osmania University. Presently, she is working as Senior Research Fellow (CSIR-UGC) at the DRDE, Gwalior, and pursuing her PhD. Her present research work is focused on development of rapid and efficient methods for the synthesis of organophosphorous compounds and its analysis.

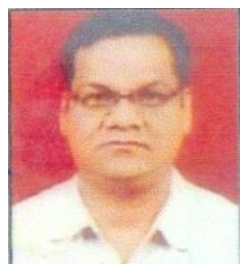

Mr Naresh Vyas, has joined DRDO in the year 2000. His area of interest is synthesis of organophosphorous compounds and analysis of the compound by GC/MS. He is also experienced in synthesis of amorphous inorganic compounds by high temperature synthetic reactions.

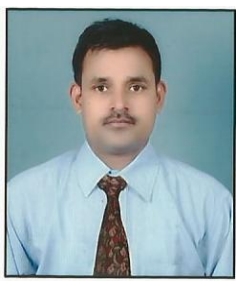

Dr. Hitendra N. Karade, did his PhD from Jiwaji University, Gwalior, MP, India. At present, he is working as scientist at Defence R\&D Establishment (DRDE), Gwalior. He is working in the area of medical protection against Organophosphorus poisoning.

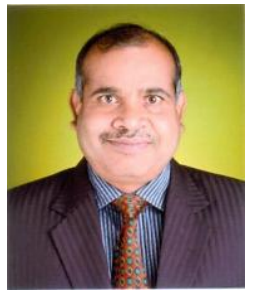

Dr A.K.Gupta, is working as a Scientist F at DRDE Gwalior. His areas of interest include synthesis of toxic agents and their characterisation. $\mathrm{He}$ is experience in preparing all type of organophosphorous compounds and their toxins. 